\title{
Effects of elevating colonic propionate on liver fat content in overweight adults with non-alcoholic fatty liver disease: a pilot study
}

\author{
E. S. Chambers ${ }^{1}$, A. Viardot ${ }^{1}$, A. Psichas ${ }^{1}$, D. J. Morrison ${ }^{2}$, K. G. Murphy ${ }^{3}$, \\ S. E. K. Zac-Varghese ${ }^{3}$, K. MacDougall ${ }^{4}$, T. Preston ${ }^{2}$, M. C. Tedford ${ }^{4}$, J. D. Bell ${ }^{5}$, \\ E. L. Thomas ${ }^{5}$, S. Mt-Isa ${ }^{6}$, D. Ashby ${ }^{6}$,W. S. Dhillo ${ }^{3}$, S. R. Bloom ${ }^{3}$, W. G. Morley ${ }^{3}$, \\ S. Clegg ${ }^{7}$ and G. Frost ${ }^{1}$ \\ ${ }^{1}$ Nutrition and Dietetic Research Group, ${ }^{3}$ Section of Investigative Medicine, Imperial College London W12 0NN, UK, \\ ${ }^{2}$ Scottish Universities Environmental Research Centre, University of Glasgow G75 0QF, UK, ${ }^{4}$ School of Science, \\ University of the West of Scotland, Paisley PA1 2BE, UK, ${ }^{5}$ MRC Clinical Science Centre, Imperial College London \\ W12 ONN, UK, ${ }^{6}$ Imperial Clinical Trials Unit, Imperial College London W2 $1 N Y$, UK and ${ }^{7}$ Leatherhead Food \\ Research, Leatherhead KT22 7RY, UK
}

It is estimated that $20-30 \%$ of adults in developed countries have non-alcoholic fatty liver disease (NAFLD), a condition characterized by the accumulation of fat within the liver ${ }^{(1)}$. NAFLD is commonly associated with an increased risk of developing Type 2 diabetes. Positive physiological effects have been linked with the production of short chain fatty acids (SCFA) by colonic fermentation of non-digestible carbohydrate (NDC), including reductions in hepatic fat content ${ }^{(2)}$. We have developed a novel inulin-propionate ester, whereby the SCFA propionate is bound to the NDC inulin. The bound propionate is only released from the inulin carrier molecule through bacterial fermentation in the colon allowing the targeted delivery of gram quantities of propionate. In humans, $\sim 90 \%$ of propionate produced in the colon is extracted from portal blood by the liver. Propionate is a known gluconeogenic substrate, and the energy to drive this anabolic pathway may derive from increased hepatic fat oxidation ${ }^{(3)}$. We hypothesised that long-term elevations in colonic propionate production would reduce liver fat in adults with NAFLD.

Sixteen participants aged 40-65 years, with a BMI of $25-40 \mathrm{~kg} / \mathrm{m} 2$ were recruited and intrahepatocellular lipid (IHCL) was determined using ${ }^{1} \mathrm{H}$ magnetic resonance spectroscopy (MRS) ${ }^{(4)}$. Participants were identified as having NAFLD on the basis of a liver fat content $>5 \cdot 5 \%{ }^{(5)}$. Participants were provided with either $10 \mathrm{~g} /$ day propionate ester $(n=11)$ or $10 \mathrm{~g} /$ day inulin control $(n=5)$ to add to their normal diet for a 24 week intervention period. After 24 weeks of dietary supplementation, measurements taken at baseline were repeated. In the propionate ester group a significant reduction in IHCL content post-intervention was observed $(22 \cdot 1 \%$ to $15 \cdot 9 \% ; P=$ 0.038). This effect was not found in the control group (19.1\% to $18.7 \% ; P=0.576)$. An improvement in liver function tests (alanine amino transferase and aspartate aminotransferase) was also observed within the propionate ester group, whilst these liver function tests were unchanged in the control group. There was no significant change in body weight following the intervention period in either group.

Our data provides the first direct evidence in humans that gut-derived propionate can reduce liver fat in adults with NAFLD. Targeting increased colonic propionate levels, delivered via enriched food products, may offer a public health solution to the increased prevalence of NAFLD at the population level and warrants further investigation.

This work was supported by the Biotechnology \& Biological Sciences Research Council (BB/H004971/1). The clinical trial (NCT00750438) was conducted in accordance with the Declaration of Helsinki.

1. Preiss D \& Sattar N. (2008) Clin Sci (Lond) 115, 141-50.

2. Anastasovska J, Arora T, Sanchez Canon GJ, et al. (2012) Obesity (Silver Spring) 20, 1016-23.

3. Sunny NE, Parks EJ, Browning JD, et al. (2011) Cell Metab 14, 804-10.

4. Thomas EL, Parkinson JR, Frost GS, et al. (2012) Obesity (Silver Spring) 20, 76-87.

5. Browning JD, Szczepaniak LS, Dobbins R, et al. (2004) Hepatology. 40, 1387-95. 